\title{
Design explanation: determining the constraints on what can be alive
}

\author{
Arno G. Wouters
}

Received: 22 March 2005/Accepted: 9 March 2007/Published online: 19 April 2007

(C) Springer Science+Business Media B.V. 2007

\begin{abstract}
This paper is concerned with reasonings that purport to explain why certain organisms have certain traits by showing that their actual design is better than contrasting designs. Biologists call such reasonings 'functional explanations'. To avoid confusion with other uses of that phrase, I call them 'design explanations'. This paper discusses the structure of design explanations and how they contribute to scientific understanding. Design explanations are contrastive and often compare real organisms to hypothetical organisms that cannot possibly exist. They are not causal but appeal to functional dependencies between an organism's different traits. These explanations point out that because an organism has certain traits (e.g., it lives on land), it cannot be alive if the trait to be explained (e.g., having lungs) were replaced by a specified alternative (e.g., having gills). They can be understood from a mechanistic point of view as revealing the constraints on what mechanisms can be alive.
\end{abstract}

Keywords Functional explanation $\cdot$ Mechanistic explanation $\cdot$ Design explanation · Constraints

\section{Introduction}

Why do fishes respire with gills rather than lungs? Why do tetrapods respire with lungs rather than gills? Why don't these organisms respire through their skin? These questions are discussed in any intermediate level textbook that deals with respiratory physiology (e.g., Johansen 1977; Dejours 1981; Withers 1992, chs. 4 \& 12; Liem et al. 2001, ch. 18; Maina 2002) and they are answered by showing that

\footnotetext{
A. G. Wouters $(\bowtie)$

Heyendaal Institute, Radboud University Nijmegen, P.O. Box 9103, 6500 HD Nijmegen,

The Netherlands

e-mail: Arno.Wouters@hin.ru.nl
} 
in the conditions that apply to the relevant organisms, the actual design is better than the contrasting design. Biologists call such reasonings 'functional explanations'. To avoid confusion with other uses of this term, I shall call them 'design explanations'. As I shall show, design explanations are contrastive, they often compare real organisms to fictitious organisms that cannot possibly exist, and they are not causal, but appeal to a kind of relation (functional dependence) that has been ignored in the philosophy of science. As I shall shortly discuss, the standard accounts of functional explanation do not apply to design explanations. In this paper I aim to provide a detailed account of the structure of design explanations and to explain how they add to scientific understanding. ${ }^{1}$ My main conclusion is that they reveal the constraints on being alive.

The question of how functional explanations contribute to scientific understanding is a long-standing issue in the philosophy of science (see Wouters 2005 for an overview). I discuss this question in view of the causal-mechanical approach to explanation. According to this approach, explanations show how a certain event, state, regularity, activity or capacity fits into the world's causal structure. This approach is best known from Salmon (1984).

Most theories of function depict functional explanations as statements of the form 'The function of $x$ is $f^{\prime}$ brought up to answer a request for an explanation of $x$ ( $x$ can be anything of a living organism: a part, a behavior, a trait, the structure of an organ, and so on). This view is nicely summarized by Andrew Woodfield in a recent encyclopedia:

Typically, a functional explanation in biology says that an organ $x$ is present in an animal [sic!] because $x$ has function $F$ (Woodfield 2000, p. 493)

The standard example concerns the heart. The idea is that biologists explain the presence of this organ simply by uttering: 'The function of the heart is to pump the blood around.'

Another important assumption is the idea that functions are a special kind of effect. An item such as the heart has many effects, but only some of them are functions.

Given this picture of functional explanations as consisting of function attributions, given the impression that functions are effects, and assuming that explanations are causal, the main question about functional explanation becomes how an appeal to an effect of a trait's presence could explain why that trait is present. The three main kinds of answers to this problem are: (1) selected effect theories, (2) systemic theories and (3) life chances theories.

These theories do not necessarily exclude each other. The dominant opinion is that biologists employ two notions of functions associated with two kinds of explanation: systemic functions, which are used to explain how a certain capacity or activity is brought about in a certain individual; and selected effect functions, which

\footnotetext{
${ }^{1}$ It is not my goal to analyze the notion of design (I aim to analyze a kind of reasoning), to analyze a preexisting notion of 'design explanation' (this notion is stipulated), or to define the phrase 'design explanation'. Rather, I aim to describe and account for the scientific value of a kind of reasoning that is ostensively defined.
} 
are used to explain why certain organisms have a certain trait by specifying the effects for which that trait was maintained in the lineage in the past.

According to selected effect theories (for example, Williams 1966; Neander 1980; Millikan 1984), function attributions explain the presence of a certain trait by specifying the effects for which that trait was selected in the past. For example, according to these theories, the statement 'The function of tetrapods' lungs is to respire' explains why tetrapods have lungs by pointing out that in tetrapods' lineage, lungs were maintained in the process of natural selection because they helped the organism to respire. This approach provides an elegant solution to the aforementioned problem of functional explanation: functions are past effects and past effects can, of course, explain current presence (see Salmon 1989, § 3.8; Mitchell 1989; Neander 1991). However, design explanations differ from functional explanations on the selected effect view (i.e., evolutionary selection explanations). First, design explanations are contrastive (a feature that is remarkably absent from all existing accounts of functional explanation), and they often contrast real organisms with merely hypothetical alternatives that never existed (evolutionary explanations appeal to past fitness differences between variants that actually did exist). Second, design explanations are concerned with synchronic relations between the different traits of an organism and the environment in which it lives, rather than with what happened in the past. So, whatever the merits of the selected effect approach, it does not account for design explanations (see Wouters 2003, Sect. 3.2).

According to systemic theories (for example, Cummins 1975, 1983; Craver 2001), function attributions inform us about how an item contributes to the workings of a complex system. Such attributions of systemic functions are an essential part of explanations that explain a complex system's behavior by describing a mechanism that produces that behavior. For example, according to systemic theories, the attribution to the lungs of the respiratory function helps to explain how the organism gets enough oxygen.

This notion of function as a contribution to an important capacity is, indeed, the central notion of function in biology. I shall call a function in this sense a 'biological role' if its description fits in the general scheme that biologists use to explain organisms' maintenance, growth and development, and the production of offspring (see Wouters 2003 for an extensive discussion of the different notions of function in biology). Explanations that explain how a certain capacity is brought about by specifying an underlying mechanism play an important role in functional biology. Biologists call such explanations 'causal explanations'. Design explanations address a different kind of question. Given a system (e.g., a gill or a lung) that performs a certain biological role (e.g., gas exchange), an explanation that specifies an underlying mechanism would relate how the capacity to perform that role is brought about by the organized activity of that system's parts (it would, for example, explain how gas exchange takes place in the gills or lungs). A design explanation, on the other hand, would point out why that biological role is performed the way it is, rather than in some other conceivable way (for example, why it is performed by a specialized organ, and why certain kinds of organisms perform this role with gills, whereas others do so with lungs). This design explanation would do so by 
specifying the features of the relevant organisms and their environment (such as the organism's size, activity and respiratory medium) that make the actual design better than the alternatives, and by elucidating why, in these circumstances, the actual design is better than those alternatives by appealing to invariant generalizations (such as Fick's law of diffusion).

A third approach, the life chances approach (Canfield 1964, 1965; Ruse 1971, 1973; Wimsatt 1972; Bigelow and Pargetter 1987), defines functions as hypothetical advantages: a trait's function is an effect of that trait's presence which makes the organism's life chances better than they would have been if that effect were lacking. For example, according to this kind of theory, it is the function of the lungs to respire because the fitness of organisms that have a lung that aids in respiration is higher than their fitness would have been if their lungs did not do so.

This account captures an important characteristic of design explanation, namely, its appeal to counterfactual comparison. It has been criticized for this because the truth of statements about the life chances of hypothetical organisms lacking a certain trait would be indeterminate (e.g., Millikan 1993). However, as counterfactual comparison is ubiquitous in functional biology, ${ }^{2}$ it should be accounted for, rather than denied. Wouters (2003, Sect. 3.1) argues that the problem of indeterminate truth value arises because the current life chances theories attempt to explicate a non-comparative notion (a function of .... is to ....) in comparative terms. This works only if there is a unique reference situation. Moreover, this way of explicating 'function' confuses two notions of function, namely, the noncomparative notion of function as biological role (a role of .... is to ....) and the comparative notion of function as biological advantage (an advantage of .... over .... is ....). The problem dissolves if (1) the comparative analysis is restricted to advantages, and (2) advantages are explicitly defined relative to a well-specified reference situation.

Wouters' analysis shows how counterfactual comparisons can have a definite truth value. However, an account of the insights such comparisons provide is still lacking. Why do biologists engage in such reasonings? What do they learn from them? Some sympathizers of life chances views have suggested that appeals to advantageous effects can causally explain why organisms have the fitness they have (Bigelow and Pargetter 1987; Mitchell 1989; Walsh 1996). However, biologists think of design explanations as providing insight into why organisms have the traits they have (e.g., why certain kinds of organisms have lungs and other kinds have gills). I argue that this insight can be accounted for if design explanations are seen as reasonings that are concerned with the constraints on what forms of organization

\footnotetext{
${ }^{2}$ See Wouters (2003) for a half dozen examples from textbooks and research papers. A quick look into any zoology textbook or journal would reveal many more, including explanations of why bats are almost exclusively nocturnal rather than diurnal (Thomson and Speakman 1999); of why emperor penguins produce calls by using two independent sound generators, rather than one (see Sturdy and Mooney 2000); of why tiger moths inform predatory bats of their unpalatability by using clicks, rather than other kinds of cues (Ratcliffe and Fullard 2005); of why nocturnal, terrestrial vertebrates have multifocal, rather than monofocal, lenses; of why multifocal eyes have split pupils rather then round ones (Malmström and Kröger 2006); and of why the mammalian inner ear has a spiral shape (rather than being stretched out) (Manoussaki et al. 2006).
} 
(i.e., designs) can be alive, rather than with the causes of how these designs came into being. ${ }^{3}$

In the next section (Sect. 2) I give some detailed examples of the reasonings that I call 'design explanation'. In Sect. 3 I specify the reasoning structure that emerges from these examples and show that it does not fit the standard view of functional explanations as function attributions that answer why-questions about something biological. This description should make it clear that the heart of a design explanation is not a function attribution, but a statement expressing a functional dependence relation: the relevant organisms' ability to live in the conditions in which they live depends on those organisms having the traits to be explained, rather than the contrastive ones (for example, if an organism exhibits a certain size and activity, it cannot be alive if its specialized respiratory organs are replaced by respiration through the skin). ${ }^{4}$ In Sect. 4 I discuss the nature of this functional dependence relation and the manner in which it is explained. I argue that functional dependencies are individual level, synchronic relations that constrain what can be alive, but do not bring about the characteristics satisfying this constraint (in other words, they are not causal). The existence of such constraints can be explained by appealing to the relevant conditions and the invariant relations ("laws") that apply in those conditions. This opens the way to an account of the scientific value of design explanations in Sect. 5. I end up by drawing some conclusions in Sect. 6.

\section{Examples}

My examples concern how vertebrates take in oxygen from, and release carbon dioxide into, the environment. This process is called 'respiration'. Adult tetrapod vertebrates (e.g., amphibians, reptiles, birds and mammals) typically respire with lungs $;^{5}$ whereas fishes typically respire with gills. ${ }^{6}$ By definition, gills are evaginated organs (i.e., outgrowths of the body) and lungs are invaginated. Fishes' gills typically consists of a minutely subdivided surface area, composed of numerous ultra fine lamellae, across which water is pumped in one direction (from the mouth over the gills to the outside). The lamellae are richly supplied with blood vessels; the distance from the water to the blood can be less than $1 \mu \mathrm{m}$. The tetrapod lung is essentially an ingrowth of the foregut. In its simplest form it is just a sac with ridges and edges that increase its surface area. More advanced lungs consists of a system of conducting and distributing tubes which divide further and further until they end in blind, thin-walled sacs (alveoli) where gas exchange occurs. Lungs are

\footnotetext{
${ }^{3}$ I do not claim that constraints can never be causal, only that constraints on what can be alive are not.

4 My account of design explanations' structure is much closer to the accounts of Hempel (1959) and Nagel (1961) than the standard accounts are. However, my account of design explanations' scientific value is very different from their account of functional explanations' scientific value. Hempel and Nagel had an inferential view of explanation; my account is mechanistic.

5 In many amphibians the skin, too, plays an important role in respiration.

${ }^{6}$ Some fish do use lungs.
} 
ventilated by pumping air in and out them (this is called 'breathing'). The flow of air in the lungs has a tidal character, with both inhalation and exhalation phases. ${ }^{7}$ In contrast with gills, gases are not actively transported over lungs' respiratory surfaces; the alveoli contain dead air through which oxygen and carbon dioxide are transported by diffusion alone.

My example concerns two questions about how organisms respire. The first is, why do so many organisms use specialized organs (such as gills or lungs), rather than their outer surface, for respiration? The second question concerns these organs' specific character: why do fishes respire with gills, and tetrapods with lungs?

In order to answer such questions biologists typically refer to Fick's law of diffusion. This is a law of physical chemistry which states that the rate of diffusion of a gas is proportional to both the surface area available for diffusion and the concentration gradient over which diffusion takes place:

$$
J=-D A \Delta C / \Delta x
$$

in which $J$ is the rate of diffusion (mole/s); $D$ the diffusion coefficient $\left(\mathrm{cm}^{2} / \mathrm{s}\right) ; A$ the surface area available for diffusion $\left(\mathrm{cm}^{2}\right) ; \Delta x$ the distance of diffusion $(\mathrm{cm}) ; \Delta C$ the concentration difference over that distance $\left(\mathrm{mole} / \mathrm{cm}^{3}\right)$. The sign indicates the direction of diffusion; it is negative here because diffusion goes from higher to lower concentrations. The diffusion coefficient depends on the nature of the diffusing gas, the medium in which diffusion takes place and the temperature. The quantity $\Delta C / \Delta x$ is the concentration gradient $\left(\mathrm{mole} / \mathrm{cm}^{4}\right)$.

The answer to the first question (why is respiration concentrated in a specialized organ?) points to the many disadvantages of using the body's entire surface for respiration. In order to reach a certain level of activity the organism must generate a certain amount of energy, and this, in turn, requires a certain amount of oxygen and generates a certain amount of carbon dioxide. From Fick's law it follows that if the organism's need for oxygen exceeds a certain level, the respiratory surface must meet certain requirements in order to assure that the diffusion rate $(J)$ is large enough to fulfill the organism's needs: (i) the distance across which the gas must diffuse $(x)$ must be small enough, (ii) the surface available for diffusion $(A)$ must be large enough, and (iii) the material across which the gas diffuses must be readily permeable to that gas ( $D$ must be large). In other words, the respiratory surface must be thin, large and permeable. This precludes the animal from using its body's entire surface for respiration: a thin skin is easily damaged, enlargements of the outer surface would disturb its streamline, and a skin that is easily permeable to oxygen and carbon dioxide is also easily permeable to water (which is a severe disadvantage both on land, and in aquatic environments whose osmotic pressure differs from the organism's). Specialized respiratory organs provide the means to solve this problem.

\footnotetext{
7 The flow in birds' lungs is virtually unidirectional. This meets the increased demand for oxygen that their flying lifestyle imposes on them. The flow in their upper respiratory tract is tidal.
} 
In order to answer the second question (why do fishes respire with gills and tetrapods with lungs?), biologists compare the physical qualities of water and air. The concentration of oxygen in air is about 30 times higher than in air-saturated water, the diffusion coefficient of oxygen is about 30,000 times higher in air than in water, water is more than 800 times as dense as air, and water is about 60 times more viscous than air. Animals that obtain oxygen from water have to solve three problems. First, due to water's low oxygen content, they need to ventilate a much larger volume than air breathers to extract the same amount of oxygen. Second, uptake of oxygen from water is very inefficient, due to both its lower oxygen content $(\Delta C / \Delta x$ is lower) and lower diffusion coefficient $(D)$. Third, because of water's higher density and viscosity, it costs much more energy to move a certain volume of water over a certain distance than to move the same volume of air over that distance. Respiration in air faces other difficulties, including the constant risk of desiccation and the problems caused by gravitation. These problems do not occur in water.

The differences in design between gills and lungs are explained by these physical differences and the different problems that "result" from them. The respiratory surface of gills $(A)$ is immensely larger than that of lungs, and gills' respiratory membranes are much thinner ( $\Delta x$ is lower). This increases oxygen uptake efficiency. The unidirectional character of the water's flow across the gills saves the costs of reversing the flow. In addition, the continuous stream of water over the respiratory surface increases the oxygen uptake because it increases the oxygen concentration at the outside of the respiratory surface. Due to the problems of gravitation, the vast increase in gills' surface area is not possible on land; the finely divided and thin lamellae would collapse against each other. The thin membranes and unidirectional flow also would increase the risk of desiccation.

Lungs are internal, which reduces evaporation and provides structural support to counteract gravity. The tidal flow of air further reduces the loss of water. Lungs would not work in an aquatic environment for several reasons. ${ }^{8}$ One is that the lungs' pumping mechanism, breathing, has much less power than gills' pumping mechanism (this is no problem for air breathers because moving air requires much less energy than moving water). Another reason is that the tidal character of air flow in the lungs greatly decreases ventilation efficiency (recall that respiration in water imposes much more demands on ventilation than does aerial respiration).

\section{The basic structure of design explanations}

As I indicated in Sect. 1 above, functional explanations are routinely viewed as answering a question of the form 'Why do certain organisms have/perform $x$ ?' with a function attribution of the form 'In those organisms the function of $x$ is to do $f$. It will be clear from my examples that design explanations' structure is much more complex than this.

\footnotetext{
${ }^{8}$ Fishes with lungs are no exception, as they use their lungs to breathe air.
} 
First, the questions design explanations address are explicitly or implicitly comparative. The first example asked why so many animals respire by means of a specialized organ rather than using the entire skin. The second example asked why aquatic vertebrates use gills rather than lungs for respiration, and why land vertebrates use lungs rather than gills. This question is a shorthand for more specific questions, such as, 'Why is the surface of fishes respiratory organ more minutely divided than that of land vertebrates?'; 'Why do land vertebrates use an internal, rather than an external, organ to respire?'; and 'Why do fishes have a unidirectional flow over their respiratory surface, whereas land vertebrates employ a tidal flow system?? ${ }^{\text {? }}$

Second, the explanations employ two kinds of function attributions, namely, functions as biological roles (the kind of function envisaged by the systemic view) and functions as biological advantages (the kind of function envisaged by the life chances view). Attributing the biological role of respiration to the lungs and the gills plays a key role in my examples' explanations. Those explanations use this attribution to explore the advantages and disadvantages of the item that performs this role having a certain character rather than another (e.g., having the character of a lung, rather than a gill). The first example details the many disadvantages of using the skin, rather than a specialized organ, for respiration. The second example details the advantages of using lungs, rather than gills, on land and gills, rather than lungs, in water.

Third, the answer consists of much more than functional attributions. In addition to specifying the biological role of the item or activity in question, and what advantages it has in performing that role the way it is performed, design explanations point out what internal and external conditions make this character advantageous, and elucidate how those conditions are related to the advantages in terms of the laws of nature. In the first example, the relevant conditions include such things as the organisms' activity, their size and how they obtain energy. In the second example, the respiratory medium is an important condition. Fick's law of diffusion plays an important role in all these examples.

In my view, a design explanation addresses the question of why certain organisms have one trait rather than another. The design explanations in my examples compare different ways in which the biological role of respiration is performed. In my first example (why a specialized organ?) the question concerns those animals that use a specialized organ to respire, and asks why they do so rather than using the entire outer surface. My second example (why gills in water and lungs in air?) is concerned with two such questions. One is about fishes that perform aquatic gas exchange, and it asks why they respire with an organ that has the specific characters of gills (such as their minutely divided lamellae and unidirectional flow), rather than with an organ that has the specific characters of lungs (such as the fact that they are internal, the existence of dead space, and the tidal character of ventilation). The other question concerns tetrapods that respire with lungs, and it

\footnotetext{
${ }^{9}$ Note that design explanations always compare the real design with a limited range of alternative designs. They never address the question of why a certain design is better than all other conceivable ones. We can always imagine better solutions (whose lack of existence has no design explanation—see Sect. 5).
} 
asks why these organisms respire with an organ with the specific characters of lungs, rather than with an organ with the specific characters of gills.

The explanation that answers this type of question:

(1) Specifies conditions that apply to the relevant organisms;

(2) States that, due to those conditions, the trait those organisms have is more useful to them than the alternative (the nature of this relation is discussed in Sect. 4 below); and

(3) Explains (2) (this part of the explanation, too, is discussed in Sect. 4 below).

In the examples above:

Animals that use a specialized organ to respire do so (rather than using their body's entire outer surface) because:

(1a) Those organisms' activity and size exceed a certain level.

(1b) They use oxygen as an electron acceptor in the metabolic pathway with which they obtain energy.

(2) If an organism's activity and size exceed the level mentioned in (1), the biological role of respiration is better performed with a specialized organ than by using its entire outer surface.

(3a) In order to reach the level of activity mentioned in (1a), an organism of the size mentioned in (1a) needs a certain amount of energy (law of physics).

(3b) To get this amount of energy the organism needs a certain supply of oxygen (the way that aerobic organisms get their energy, see 1b).

(3c) In order to satisfy this need the respiratory surface must be thin, large and permeable (follows from Fick's law).

(3d) A skin that satisfies these conditions would have the disadvantages of being fragile (material physics), lacking a streamline (hydrodynamics) and suffering from osmotic problems (physical chemistry) and/or desiccation (physics). These problems are avoided if a specialized organ is used.

Fishes that employ aquatic gas exchange use gills rather than lungs for respiration because:

(1a) They take their oxygen from water.

(1b) They are moderately active and their size is within certain limits.

(1c) They use oxygen as an electron acceptor to generate organic energy.

(2) If the respiratory medium is water and the fishes' level of activity and size are as mentioned in $1 \mathrm{~b}$, the biological role of respiration is better performed with gills than with lungs.

(3a) Given the activity and size mentioned in $1 \mathrm{~b}$ the organism needs a certain supply of oxygen (laws of physics and way that aerobic organisms get their energy).

(3b) Due to water's low oxygen content and very small diffusion coefficient, the organism must ventilate huge amounts of water in order to get enough oxygen (arithmetic + Fick's law). 
(3c) Due to water's density and viscosity, much power is needed to move this amount of water (physics).

(3d) Because of their smaller respiratory area, their larger distance between the medium and the blood, the dead air in their alveoli and the tidal character of their air flow, lungs are less efficient than gills (Fick's law). This means that if fishes were to use lungs to take up oxygen from water, they would need even more power than they do now. The lungs' ventilation mechanism is less powerful than that of gills (the way lungs and gills are built) and does not have enough power to move the required amount of water.

(3e) Due to their large respiratory area, their small distance between the medium and the blood, and their continuous, unidirectional flow over the respiratory surface, gills are more efficient than lungs (Fick's law). In addition they have much more power (the way lungs and gills are built). As a result they are able to provide the required amount of oxygen at the required rate.

Tetrapods that respire with lungs do so rather than with gills because:

(1) They live on land and perform a certain level of activity, their size is within certain limits, they use oxygen as an electron acceptor to generate organic energy and they take their oxygen from the air.

(2) If an animal lives on land and its respiratory medium is air, the biological role of respiration is better performed with lungs than with gills.

(3a) Animals that live on land and/or breathe air are subject to gravitation (physics).

(3b) Animals that breathe air lose water via their respiratory surface (physics).

(3c) Due to gravitation, gills would collapse on land (physics); the resulting decrease in respiratory surface would kill the animal (aerobic metabolism, certain activity).

(3d) Furthermore, air breathing animals with a unidirectional stream over a thin respiratory surface would dry out (physics).

(3e) Lungs are internal. This reduces the loss of water via the respiratory surface (physics) and allows for structural support that prevents the respiratory surface's collapse (geometry). This solves the problems of gravitation and desiccation.

\section{Functional dependencies and their explanation}

The heart of a design explanation is a statement (2) that says that due to certain conditions, a trait that an organism actually has is more useful to that organism than a conceivable alternative. The notion of utility or biological value refers to an organism's ability to stay alive (i.e., to maintain itself, to grow, to develop and to produce offspring). Roughly speaking, a certain trait (e.g., using a specialized organ for respiration) is more useful to a certain organism than is another trait (e.g., using its entire outer surface for respiration), if the real organism's ability to stay alive is higher than that of a hypothetical organism in which the actual trait is replaced by 
the alternative. In many cases, the hypothetical organism cannot live. In such cases it is said that the actual trait 'is needed' by the real organism (in the relevant circumstances).

Statement (2) above says that the difference between the real and the hypothetical organisms' ability to stay alive 'is due to' certain conditions. I call the kind of relation that exists between the conditions and the trait to be explained 'functional dependence relations'. Biologists often express this kind of relation by saying that the conditions 'demand for' the organism to have the actual trait rather than the alternative. If the condition is another of the organism's traits it is often said that this latter trait 'depends on' the trait to be explained (e.g., living on land depends on using lungs (rather than gills) to respire). Phrases such as 'is due to', 'depend on', and 'demand for' indicate that the conditions are causally relevant to the utility of the trait to be explained. Roughly speaking 'causally relevant' means that if the conditions did not apply, the hypothetical organism's ability to stay alive would not be less than that of the actual one.

Functional dependence relations are individual level relations in the sense that they relate different traits of the same individual (e.g., an animal that lives on land needs lungs). They are synchronic in the sense that the need must be satisfied at the time that the demand arises (an organism that has the need but cannot satisfy it is not viable). This is an important difference between design explanations and evolutionary explanations. The latter appeal to historical relations between different individuals (that is, at the level of the lineage); in an evolutionary explanation it is the past performance of ancestral individuals with a certain trait that explains the presence of that trait in current individuals.

The nature of functional dependence relations is different from the nature of relations that figure in causal explanations. Woodward (1997) argues that the kinds of generalizations that figure in causal explanations are generalizations that are invariant under at least some (hypothetical) interventions that change the conditions described in the antecedent. Following Glennan (2000, p. S344), I call such generalizations 'invariant, change-relating generalizations'. Invariant, changerelating generalizations are weaker than the kind of generalizations that philosophers call laws, and stronger than accidental generalizations (such as the generalization that all the coins in my pocket are made of copper) and common cause generalizations (such as the relation between the barometer's reading and the weather).

Statements expressing functional dependencies are not invariant, change-relating generalizations; the presence of the needed trait is not invariant under interventions on the dependent trait. For example, although (given a certain size and activity) living on land depends on the presence of lungs and living in water depends on the presence of gills, we cannot change a dog's lungs into gills by forcing that dog to breathe underwater. Yet, unlike accidental generalities and like causal relations, functional dependencies are in a sense physically necessary: an organism that has the dependent trait cannot be alive (or will be less viable) if it has the alternative trait instead of the needed one. In other words, a functional dependency is a constraint on what can be alive. 
Unlike accidental generalizations and like causal regularities, functional dependencies can be explained on the basis of invariant relations. This is what part (3) of a design explanation does. Part (3) (I) specifies a structure of functional dependencies that relate the conditions under (1) to the utility of having the trait to be explained, and (II) relates this structure of functional dependencies to invariant relations that result from the way the organism is wired, and from more general invariant relations that scientists call 'physical laws' (in the schemes of reasoning at the end of Sect. 3 above I have indicated part (II) between brackets).

For example, part I of the explanation of why so many organisms use a special organ to respire specifies that the organisms' level of activity and size depend on their ability to generate a certain amount of energy (3a). This, in turn, depends on a certain supply of oxygen ( $3 b)$. This, in turn, demands that the respiratory surface be thin, large and permeable (3c); which, in turn, demands a specialized organ (3d). Part II relates each of these dependencies to invariant, change-relating relations that apply to the relevant organisms in the conditions mentioned in (1). The explanation of the functional dependence of activity and size on a certain level of energy production ( $3 \mathrm{a}$ ) points out that (i) there is an invariant relation between body mass and energy consumption (energy consumption increases with activity and body mass), and (ii) energy consumption can never be larger than energy production. The explanation of energy production's functional dependence on oxygen supply (3b) points out that (i) there is an invariant relation between energy production and oxygen consumption (the production of a certain amount of energy uses a certain amount of oxygen), and (ii) the oxygen supply must meet the oxygen consumption. The explanation of the oxygen supply's dependence on the respiratory surface being thin, large and permeable (3c) employs Fick's law: the only combination that satisfies the oxygen need of a large and active organism is one in which $\Delta x$ is small, $A$ is large and $D$ is small. The final step (3d) aims to show that the hypothetical organism with the alternative trait would not satisfy its needs as well as the real organism does. This can be done directly, but the example does it by means of an advantage articulation. The explanation states at this point that an organism that satisfies the need for a large, thin and permeable respiratory surface and uses its skin to respire will suffer some disadvantages that the solution in the real organism does not present; e.g., its skin will be easily damaged, the organism will lack streamline and may have problems with osmoregulation and desiccation. The disadvantages of this implementation are calculated with the help of invariant generalizations.

The invariant, change-relating generalizations used in (3II) to explain the functional dependencies of (3I) need explaining. This is done by describing the mechanism underlying this relation (see Glennan 2000). The process of providing deeper explanations ends if the explanation of a certain generalization is considered to be part of another discipline or if the explanation is registered as a yet unsolved problem. For example, the explanation of the precise relation between body mass and energy needs (3ai) is a major unsolved problem in animal physiology. It is known from physics that the energy need increases with activity and body mass. However, the relation between body mass and energy need is not linear as might be expected at first. Measurements show that energy needs are proportional to body mass by a factor of (about) 0.75 (this factor is called the mass exponent), but it is 
unclear why this is the case. The explanation of the relation between the needs for energy and oxygen (3bii), however, is well understood. This relation is determined by the metabolic pathways involved in the production of organic energy. Knowledge of the relevant pathways allows us to calculate the oxygen need. The explanation of Fick's law (3c) is a matter of physical chemistry, which biologists simply take as fundamental.

\section{How design explanations contribute to scientific understanding}

According to the causal-mechanical view of explanation, explanations reveal the mechanisms that produce the event or regularity to be explained. This means that the main insight produced by a causal-mechanical explanation is the insight into how (i.e., by means of what causes and mechanisms) an event or regularity is brought about. However, as I have emphasized in the preceding section, design explanations are not causal. The functional dependence relation that is central to design explanations (for example, the relation between an organism's activity and size and its use of a special organ for respiration, or the relation between the environment (water/land) and the character of the respiratory organ (gills/lungs)) does not vary under interventions on the related variables, and it provides no insight into how the respiratory organs are brought about (neither in the course of individual development, nor in the course of evolution).

To understand what design explanations add to scientific understanding, it should be noted that the state of being alive critically depends on the individual's organization, that is, on the spatial arrangements of its parts and the timing of their activities (see Wimsatt 1997; Craver 2001) for this notion of organization). As the diversity of living organisms testifies, there are many ways ('forms of organization' or 'designs') to construct mechanisms that are alive. However, not all arbitrary combinations of parts result in a living mechanism. Certain ways of life (e.g., living an active life on land) are possible only if certain constraints are satisfied (e.g., the problems of desiccation and gravitation must be solved). This makes it possible to explain the structure and activity of a living mechanism's parts on the basis of the constraints imposed on it by other characteristics of this mechanism and the conditions under which it works. This is what design explanations do.

Design explanations explain the structure and activity of a certain part of a living mechanism by (i) showing that this mechanism would not work as well as it does if this part or behavior were replaced by one with other characteristics (design explanations show that this part or behavior is useful or needed), (ii) specifying other traits of the mechanism that give rise to this constraint on being alive (design explanations specify which traits functionally depend on the part or behavior to be explained), and (iii) describing how this functional dependency results from the conditions and invariant, change-relating relations that apply to this mechanism (design explanations tell us why this dependency holds). As a result it becomes clear why the mechanism consists of the parts of which it consists and why those parts are organized the way they are in the context of the overall design of the organism. 
Some readers might object that, precisely because the reasonings that I have called 'design explanations' do not give insight into the causes of the phenomenon to be explained, they do not deserve to be called 'explanations'. For example, in the view of Mahner and Bunge (2001), explanation is by definition causal. To show that a certain structure or activity is useful is "a description or perhaps even a subsumption, but not a mechanismic explanation' (p. 83). I have no opinion about the answer to the question of whether or not design explanations really are explanations. In fact, I don't see that this question makes any sense at all. However, it should be noted that design explanations do not merely show that a certain structure is useful, but also why it is so. The latter is done, not merely by subsuming it under a functional dependence relation, but also by explaining why this relation holds. In this respect, design explanations do not differ from mechanistic explanations of causal regularities such as the explanation of the general gas law by statistical mechanics.

Like the explanation of the periodic table of elements in chemistry and the explanation of Kepler's laws by Newtonian mechanics, design explanations are concerned with what can possibly exist and what cannot. Design explanations seek to explain why an organism with the trait to be explained can be alive whereas a similar organism in which that trait is replaced by another one cannot. No principles restrict the kinds of contrasts for which we might want to seek design explanations. Note, however, that not all contrastive why-questions have a design answer. If the alternative trait is more useful than or functionally equivalent to the real trait (why are our bones white rather than purple?), a design explanation of why certain organisms have that trait rather than the alternative cannot be given. This is simply not the kind of question a design explanation can address. It is an empirical issue (rather than a philosophical one) which particular contrasts and how many contrasts can be explained by a design explanation.

There are also many questions for which a design answer would be irrelevant. There are three types of limitations on living beings' design: (1) it must be possible to generate individuals with that design, (2) once generated, a design must be viable (the organisms having that design must be able to stay alive), and (3) there must be an evolutionary path to that design. Design explanations are concerned with only the second kind of limitation. Clearly, if there is reason to assume that a certain design cannot be generated (how to supply a freely rotating wheel with blood vessels?), consideration of its ability to stay alive would be a waste of energy, time and money. Similarly, if we have reason to assume that no viable evolutionary path can connect an alternative design to (past or current) living forms, there would be no need to seek a design explanation.

\section{Conclusion}

Functional biologists devote a large part of their time to the construction of design explanations. Up to now there was no satisfactory philosophical account of what those explanations add to science. This paper sketches how such an account can be given within a mechanistic view of explanation. Design explanations address the 
question of why it is more useful to certain organisms to have a trait they have rather than some conceivable alternative. They answer this question by appealing to the constraints on being alive set by other traits of the organism and its environment. The explanation details those traits and shows that due to those other traits, the organism would be worse off if the trait to be explained were replaced by the alternative. The existence of this functional dependency in its turn is explained by specifying a structure of intermediate functional dependencies that relate the explaining traits to the traits to be explained. Each dependency in this structure is subsequently explained in terms of invariant, change-relating relations (some of them result from how the organism is built; others are more general). In doing so, design explanations provide both an understanding of why a certain trait is needed in the context of the overall design of a living mechanism, and an insight into the constraints on the construction of living mechanisms.

Acknowledgements Earlier versions of this paper were presented at the ISHPSSB meeting in Quinnipiac (June 2001) and at the Séminaire de la Philosophie de la Biologie of the IHPST in Paris (December 2005). The author thanks Bob Richardson, Fred Boogerd, Stuart Glennan, Françoise Longy, Michel Morange, Jean Gayon, Philippe Huneman and several anonymous referees for their useful comments.

\section{References}

Bigelow, J., \& Pargetter, R. (1987). Functions. Journal of Philosophy, 84, 181-196.

Canfield, J. (1964). Teleological explanation in biology. British Journal for the Philosophy of Science, 14, 285-295.

Canfield, J. (1965). Teleological explanation in biology: A reply. British Journal for the Philosophy of Science, 15, 327-331.

Craver, C. F. (2001). Role functions, mechanisms, and hierarchy. Philosophy of Science, 68, 53-74.

Cummins, R. (1975). Functional analysis. Journal of Philosophy, 72, 741-765.

Cummins, R. (1983). The nature of psychological explanation. Cambridge: The MIT Press.

Dejours, P. (1981). Principles of comparative respiratory physiology. Amsterdam: Elsevier/NorthHolland Biomedical Press.

Glennan, S. S. (2000). Rethinking mechanistic explanation. Philosophy of Science, 69, S342-353.

Hempel, C. G. (1959). The logic of functional analysis. In L. Gross (Ed.), Symposium on sociological theory (pp. 271-287). New York: Harper and Row.

Johansen, K. (1977). Respiration and circulation. In A. B. Kluge (Ed.), Chordate structure and function (pp. 306-391). New York: Macmillan.

Liem, K. F., Bemis, W. E., Walker Jr. W. F., \& Grande, L. (2001). Functional anatomy of the vertebrates. Fort Worth: Harcourt.

Mahner, M., \& Bunge, M. (2001). Function and functionalism: A synthetic perspective. Philosophy of Science, 68, 75-49.

Maina, J. N. (2002). Functional morphology of the vertebrate respiratory systems. Enfield: Science Publishers.

Malmström, T., \& Kröger, R. H. H. (2006). Pupil shapes and lens optics in the eyes of terrestrial vertebrates. Journal of Experimental Biology, 209, 18-25.

Manoussaki, D., Dimitriadis, E. K., \& Chadwick R. S. (2006). Cochlea's graded curvature effect on low frequency waves. Physical Review Letters, 96, 088701.

Millikan, R. G. (1984). Language, thought, and other biological categories. Cambridge: MIT Press.

Millikan, R. G. (1993). Propensities, exaptations, and the brain. In White queen psychology and other essays for Alice (pp. 31-50). Cambridge: The MIT Press.

Mitchell, S. D. (1989). The causal background of functional explanation. International Studies in the Philosophy of Science, 3, 213-230. 
Nagel, E. (1961). The structure of science. London: Routledge \& Kegan Paul.

Neander, K. (1980). Teleology in biology. Paper presented to the AAP conference in 1980.

Neander, K. (1991). The teleological notion of 'Function'. Australian Journal of Philosophy, 69, 454-468.

Ratcliffe, J. M., \& Fullard, J. H. (2005). The adaptive function of tiger moth clicks against echolocating bats. Journal of Experimental Biology, 208, 4689-4698.

Ruse, M. (1971). Functional statements in biology. Philosophy of Science, 38, 87-95.

Ruse, M. (1973). The philosophy of biology. London: Hutchinson.

Salmon, W. C. (1984). Scientific explanation and the causal structure of the world. Princeton: Princeton University Press.

Salmon, W. C. (1989). Four decades of scientific explanation. Minneapolis: University of Minnesota Press.

Sturdy, C. B., \& Mooney, R. (2000). Two voices are better than one. Current Biology, 10, R634-R636.

Thomson, S. C., \& Speakman, J. R. (1999). Absorption of visible spectrum radiation by the wing membranes of living pteropodid bats? Journal of Comparative Physiology B, 169, 187-194.

Walsh, D. M. (1996). Fitness and function. British Journal for the Philosophy of Science, 47, 553-574.

Williams, G. C. (1966). Adaptation and natural selection. Princeton: Princeton University Press.

Wimsatt, W. C. (1972). Teleology and the logical structure of function statements. Studies in History and Philosophy of Science, 3, 1-80.

Wimsatt, W. C. (1997). Aggregativity: Reductive heuristics for finding emergence. Philosophy of Science, 64, S372-S384.

Withers, P. C. (1992). Comparative animal physiology. Fort Worth: Saunders.

Woodfield, A. (2000). Teleological explanation. In W. H. Newton-Smith (Ed.), A companion to the philosophy of science (pp. 492-494). Oxford: Blackwell.

Woodward, J. (1997). Explanation, invariance and intervention. Philosophy of Science, 64, S26-S41.

Wouters, A. G. (2003). Four notions of biological function. Studies in History and Philosophy of Biology and Biomedical Science, 34, 633-668.

Wouters, A. G. (2005). The function debate in philosophy. Acta Biotheoretica, 53, 123-151. 